\title{
Reply to Letter to Editor RE: Laparoscopic Sleeve Gastrectomy After Endoscopic Sleeve Gastroplasty: Technical Aspects and Short-Term Outcomes
}

\author{
Aayed R. Alqahtani ${ }^{1,2}$ (D) \\ Published online: 5 November 2019 \\ (C) Springer Science+Business Media, LLC, part of Springer Nature 2019
}

We thank the authors of this letter for expressing interest in our study on laparoscopic sleeve gastrectomy (LSG) after endoscopic sleeve gastroplasty (ESG) [1]. We share their views on endoscopic bariatric therapy (EBT). We also agree that ESG fills a gap between medical management of obesity and bariatric surgery [2]. The procedure serves an unmet need in the care of patients with obesity, including those who are not eligible for or do not desire bariatric surgery, or as a bridge to surgery.

While acknowledging the gross similarities between ESG and LSG, we believe that it is critical not to equate the procedures with each other. Each has its own indication, weight loss mechanism, and adverse event profile. LSG is not purely restrictive. Multiple studies documented neurohumoral and metabolic changes after LSG [3-5]. While both procedures alter gastric emptying and lower ghrelin levels [6], the metabolic changes that are observed after LSG are more pronounced. Additionally, emerging results on long-term weight loss after sleeve gastrectomy suggest that success is durable $[7,8]$. Sleeve gastrectomy therefore remains a feasible revision option.

We agree that patients who are counseled on undergoing an EBT procedure should receive rigorous evaluation in order to aid in the development of a personalized bariatric therapeutic approach. Additionally, this evaluation would help in identifying and targeting eating disorders and predict which patient benefits the most from which procedure.

Aayed R. Alqahtani

qahtani@yahoo.com

New You Medical Center, Riyadh, Saudi Arabia

2 Department of Surgery, College of Medicine, King Saud University, Riyadh, Saudi Arabia
We are in urgent need for EBT guidelines that cover all aspects of this emerging approach including indications, perioperative protocol, follow-up, and revision.

\section{References}

1. Alqahtani AR, Elahmedi M, Alqahtani YA, et al. Laparoscopic sleeve gastrectomy after endoscopic sleeve gastroplasty: technical aspects and short-term outcomes. Obes Surg. 2019. https://doi.org/ 10.1007/s11695-019-04024-x.

2. Alqahtani A, Al-Darwish A, Mahmoud AE, Alqahtani YA, Elahmedi M. Short-term outcomes of endoscopic sleeve gastroplasty in 1000 consecutive patients. Gastrointest Endosc. 2019;89(6):1132-1138.

3. Abdeen GN, Miras AD, Alqahtani AR, et al. Vertical sleeve gastrectomy in adolescents reduces the appetitive reward value of a sweet and fatty reinforcer in a progressive ratio task. Surg Obes Relat Dis. 2019;15(2):194-9.

4. Madsbad S, Dirksen C, Holst JJ. Mechanisms of changes in glucose metabolism and bodyweight after bariatric surgery. Lancet Diabetes Endocrinol. 2014;2(2):152-64.

5. Ryan KK, Tremaroli V, Clemmensen C, et al. FXR is a molecular target for the effects of vertical sleeve gastrectomy. Nature. 2014;509(7499):183-8.

6. Abu Dayyeh BK, Acosta A, Camilleri M, et al. Endoscopic sleeve gastroplasty alters gastric physiology and induces loss of body weight in obese individuals. Clin Gastroenterol Hepatol. 2017;15(1):37-43.e1.

7. Puzziferri N, Roshek 3rd TB, Mayo HG, et al. Long-term follow-up after bariatric surgery: a systematic review. JAMA. 2014;312(9): 934-42.

8. Switzer NJ, Prasad S, Debru E, et al. Sleeve gastrectomy and type 2 diabetes mellitus: a systematic review of long-term outcomes. Obes Surg. 2016;26(7):1616-21.

Publisher's Note Springer Nature remains neutral with regard to jurisdictional claims in published maps and institutional affiliations. 